\title{
Information Search in Decisions From Experience: Do Our Patterns of Sampling Foreshadow Our Decisions?
}

Psychological Science $21($ I2) I787-I792 (c) The Author(s) 2010 Reprints and permission: sagepub.com/journalsPermissions.nav DOI: $10.1177 / 0956797610387443$ http://pss.sagepub.com @SAGE

\section{Thomas T. Hills and Ralph Hertwig}

University of Basel

\begin{abstract}
Do different patterns of sampling influence the decisions people make, even when the information the decisions are based on is equivalent? Do more and less switching between options correlate with different kinds of decision policies? In past research, the correspondence between search and decision patterns has been difficult to ascertain because the information obtained has often been confounded with its consequences in an exploration-exploitation trade-off. We used a sampling task in which information is explored prior to being exploited. We found that search patterns did reveal decision policies. Individuals who transitioned more frequently between options were more likely to choose options that win most of the time in round-wise comparisons and were more likely to underweight rare, risky events. Less switching between options was associated with choosing options that win in the long run on the basis of summary comparisons-decisions consistent with expected-value maximization and linear weighting of outcomes.
\end{abstract}

\section{Keywords}

decisions from experience, underweighting rare events, exploration, exploitation

Received 5/28/10; Revision accepted 7/15/10

In the year 1611, Barbara, first wife of the imperial mathematician and court astronomer Johannes Kepler, died of cholera in Prague. Kepler, widower and father of two, immediately began a methodical quest for a replacement. He considered 11 candidates, eventually choosing Susanna Reuttinger, who, he wrote, "won me over with love, humble loyalty, economy of household, diligence, and the love she gave the stepchildren" (Connor, 2004, p. 252). Although we do not know how he inspected the 11 candidates, several search strategies are possible: Antedating modern online dating strategies, he could have chatted with each of them on alternate days over a period of months, recording whom he liked most over each series of 11 days. Alternatively, he could have spent weeks at a time with each candidate, making summary assessments of each.

Although both search strategies could uncover the same information, the choices that follow from them would not necessarily be the same. The first strategy might have led Kepler to choose the person who was better in more of the 11-day bouts than any other candidate. The second strategy, in contrast, might have led him to choose a partner whose long-term mate value turned out to be best. This could have been a person who was not the best companion on many days of the year but who greatly surpassed any competitor on a few days. This divergence in the final decision highlights an important possibility: Specific sequential search strategies employed in making a choice could be coupled with specific decision strategies employed to render the final decision. This coupling was the focus of our study.

We examined this possible coupling by focusing on a paradigm that has been termed decisions from experience (Hertwig, Barron, Weber, \& Erev, 2004) in the study of choice under risk. One task used to study such decisions is the sampling task (Hertwig \& Erev, 2009): People sample as long as they wish from two initially unknown payoff distributions before making a final exploitative draw. Sampling involves two elements. First, participants choose one of the distributions - one distribution could, for instance, offer \$32 with a probability of $10 \%$, or $\$ 0$ otherwise; the other distribution could offer \$3 for sure. Second, participants witness the outcomes according to each distribution's associated payoff schedules (e.g., \$0, $\$ 0, \$ 32$ in the first distribution and $\$ 3, \$ 3$,

\section{Corresponding Author:}

Thomas T. Hills, University of Basel, Department of Psychology,

Missionsstrasse 60/64, 4055 Basel, Switzerland

E-mail: thomas.hills@unibas.ch 
$\$ 3$ in the second). Thus, in this paradigm, participants can experience as many outcomes as they wish without the associated monetary consequences. Once the evaluation is terminated, participants make a final, consequential choice.

Experienced-based risky decisions can deviate systematically from decisions from descriptions (Hertwig et al., 2004) - a phenomenon called the description-experience gap. People make description-based decisions when presented with options in which all outcomes and probabilities are stated explicitly - for instance, "\$32 with a probability of $10 \%$ and $\$ 0$ otherwise versus $\$ 3$ with certainty." In decisions from experience, people often behave as if rare events (e.g., winning \$32) have less impact than they deserve according to their objective probabilities. In decisions from description, however, people behave as if the rare events have more impact than they deserve, and they therefore make decisions consistent with prospect theory (Kahneman \& Tversky, 1979).

\section{Is the Description-Experience Gap Related to How People Search?}

Hertwig and Erev (2009) reviewed possible causes of the description-experience gap. These causes included sample size (people may take samples in quantities too small to yield rare events), recency (people may overweight recent, often common, events), estimation error (people may underestimate the frequency of rare events), and format-dependent cognitive algorithms (the different formats of statistical information in decisions from experience and decisions from description may trigger different cognitive algorithms).

There is, however, another possible cause that has previously been ignored. Could it be that the description-experience gap is related to people's sampling strategies and the potentially corresponding decision policies that people employ? Figure 1a depicts the two paradigmatic sequential-sampling strategies that we illustrated with Kepler's mate search. Given two options, at least two strategies can be used to explore them sequentially. In piecewise sampling, a search oscillates between options, each time drawing the smallest possible sample. In comprehensive sampling, a search samples extensively from one option and then samples extensively from the other option. Taking these two sampling strategies as a starting point-acknowledging that many strategies will fall on the continuum between them - we hypothesized that the way people sample is related to how they make their final decision.

Specifically, we suggest that individuals who sample piecewise will be more likely to make decisions consistent with a round-wise decision strategy: determining which option is better in each round of sampling and ultimately choosing the one that wins the most rounds (Fig. 1b, left). In comparison, individuals who use a comprehensive sampling strategy will be more likely to make decisions consistent with a summary decision strategy: evaluating average yields and then choosing the option with the larger average yield (Fig. 1b, right). The reason is that the different search strategies facilitate comparisons a

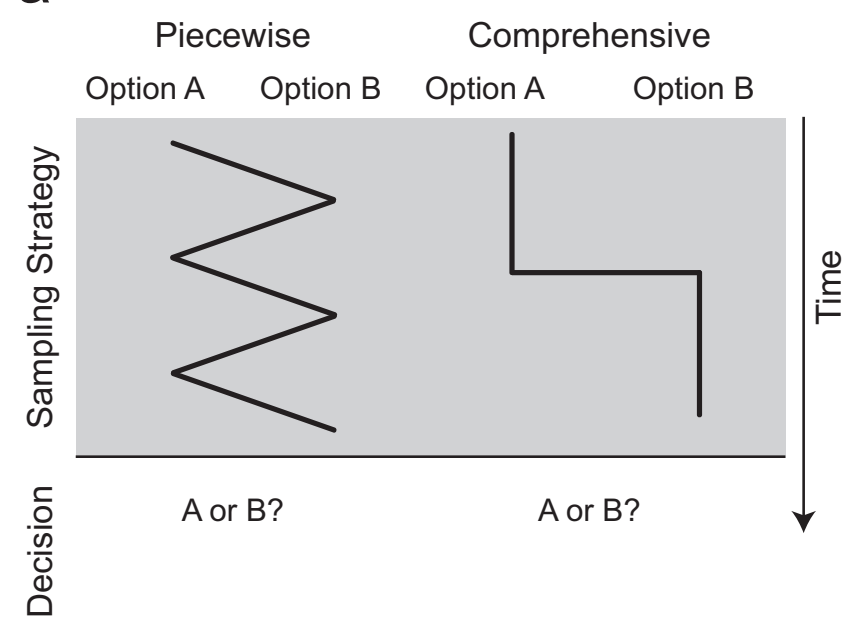

b

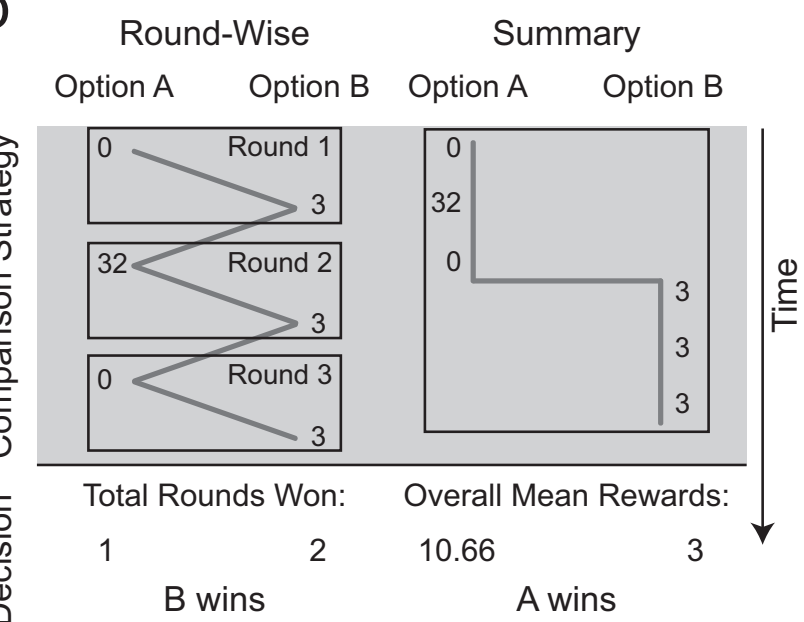

Fig. I. Two theoretical sampling patterns (a) and associated decision strategies (b). Piecewise sampling strategies alternate back and forth between options, whereas comprehensive sampling strategies take one large sample from each option in turn. Following the sampling phase, participants make a decision about which option they prefer. Round-wise decision strategies compare outcomes over repeated rounds and lead strategists to choose options that win the most rounds. Summary decision strategies compare final mean values (here, the overall expected value) and lead strategists to choose options with the higher final value.

across different scales of information (i.e., rounds compared with summaries). As Figure $1 \mathrm{~b}$ illustrates, these decision strategies can lead to different choices even though they process the same information. ${ }^{1}$

Could such a coupling between search strategies and decision strategies help explain the emergence of the descriptionexperience gap? If frequent switching between options is associated with a round-wise decision strategy, then the answer is yes, because this strategy weighs each round equally, ignores the magnitude of wins and losses, and thus underweights rare outcomes. If this line of reasoning holds, we should observe two dependencies in decisions from experience. First, there should be a dependency between people's sampling strategies 
and their decision strategies - with more switching between options corresponding to more decisions consistent with round-wise comparisons. Second, there should be a dependency between decision strategies and the psychological impact of rare events - with more switching between options (and round-wise comparisons) corresponding to more underweighting of rare events. Finding evidence for these dependencies would require a large set of data because the sampling strategies and decision strategies depicted in Figure 1 are idealizations; in reality, there are many variants of them. To assemble a large data set, we took advantage of the fact that in recent years, several studies have investigated decisions from experience using very similar methodologies.

\section{Method}

We pooled decisions from experience and search data from the following four published studies: Hertwig et al. (2004); Experiments 1 and 2 of Hau, Pleskac, Kiefer, and Hertwig (2008); Ungemach, Chater, and Stewart (2009); and Hertwig and Pleskac (2010). ${ }^{2}$ All data from these studies stem from freesampling conditions, in which participants chose freely how long to sample and how to distribute their search over two options. Because we were not interested in underweighting as a result of small sample sizes (see Hertwig et al., 2004), we included only those decisions in which participants encountered all outcomes for each option at least once in their sample. The final data set encompassed 1,223 decisions over 233 participants.

Across all studies, the experimental procedure was the same: Participants saw two buttons on a computer screen and were told that each button was associated with a payoff distribution. Clicking on a button revealed an outcome from the respective payoff distribution. Participants' search policies were recorded as they sampled - until they indicated their final decision.

\section{Results}

We first analyzed participants' switching behavior by calculating the ratio between a person's switches and the maximum number of allowable switches (i.e., $n-1$, with $n$ being the total number of draws). People's propensity to switch ranged widely, from a minimum ratio of .02 to a maximum ratio of .95 (Fig. 2). In accordance with the notion that there may be two types of sampling strategies, the distribution was bimodal, with peaks near.1 and .5. This distribution was well split by the median of .16 (indicated by the dotted line in Fig. 2). We defined participants above the median as frequent switchers and participants below the median as infrequent switchers.

\section{Is there a dependency between sampling and deciding?}

For each participant, we simulated the final decision predicted by both the round-wise and the summary decision strategies. We focused on only those decisions for which the round-wise strategy and the summary strategy predicted different final choices-leaving us with 59 frequent switchers and 55 infrequent switchers. People who switched between options more often had a significantly larger proportion of their decisions predicted by the round-wise strategy (Fig. 3). In contrast, infrequent switchers' decisions were better predicted by the summary strategy, $t(112)=-3.22, p<.01$. There was thus a clear dependency between switching during sampling and the final decision.

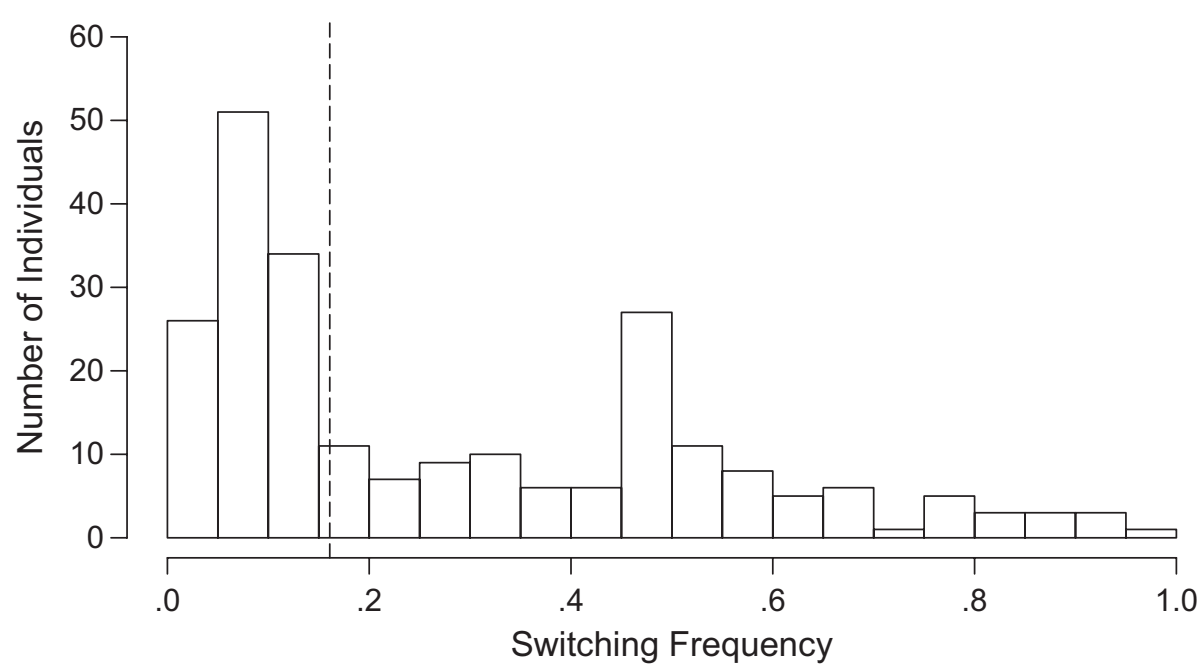

Fig. 2. Histogram of people's propensity to switch between two options. The observed number of switches is expressed as the ratio of the observed switches over the maximum number of allowable switches. For each of the 233 participants, we calculated the mean switching frequency across problems. The dotted line represents the median value of .16 . 


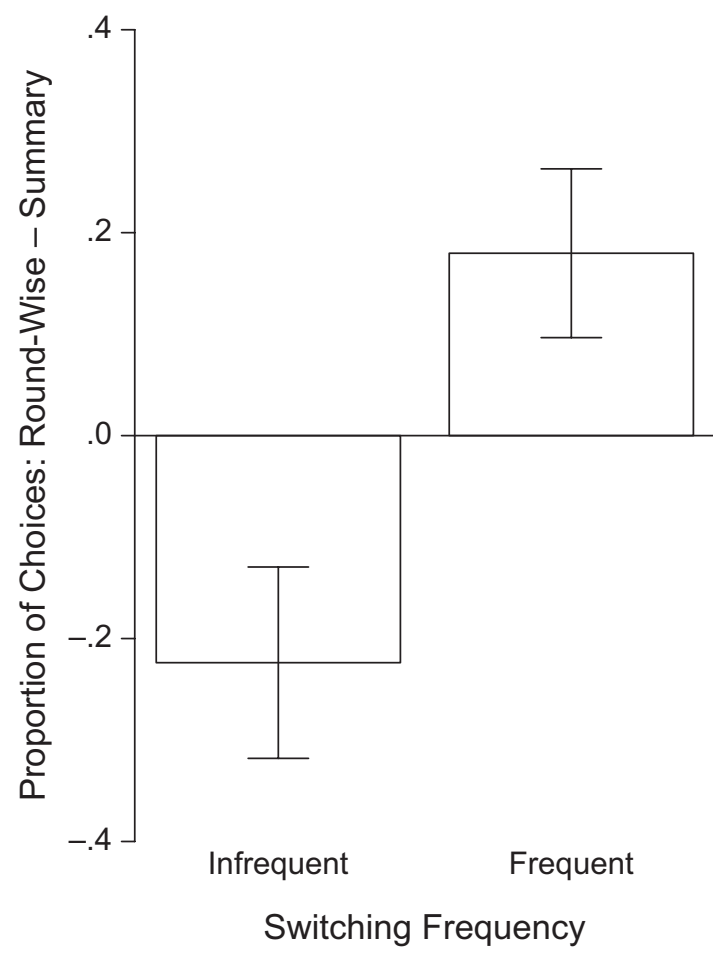

Fig. 3. Differences in the proportion of decisions that were consistent with the round-wise strategy and the summary strategy as a function of the frequency of switching between two options. A positive difference means that more choices were consistent with the round-wise decision strategy than with the summary decision strategy; a negative difference indicates a preponderance of choices consistent with the summary strategy. Error bars represent standard errors of the mean.

Could sampling effort (i.e., sample size) also predict the use of a round-wise strategy? The results of Rakow, Demes, and Newell (2008) suggested a potential correlation between sample size and switching frequency. Our results confirmed such a relationship. Using only problems in which all outcomes were seen, we found that switching frequency was negatively correlated with the total number of samples, $r=-.44, t(231)=-7.4$, $p<.001$. However, using a median split to divide the sample sizes, we found that sample size did not have a significant effect on the frequency of usage of the round-wise strategy ( $p=$ .15). Thus, frequency of switching appears to be a better predictor of round-wise decision policies than does sample size. ${ }^{3}$

\section{Is there a dependency between sampling, deciding, and the impact of rare events?}

To investigate the relationship between decision strategy and the impact of rare events, we calculated how often decisions based on the round-wise strategy or the summary strategy would underweight rare events across the 1,223 decisions. (We computed both strategies for each participant's sampling data.) For instance, in the decision problem that offered $\$ 32$ with a chance of $10 \%$ ( $\$ 0$ otherwise) or $\$ 3$ for sure, choosing the $\$ 3$ for sure would be consistent with underweighting the rare $\$ 32$ event. Figure 4 shows that if participants were using the roundwise strategy, they would have been significantly more likely to underweight the rare event than if they were using the summary strategy, paired $t(231)=-7.39, p<.001$. As predicted, the strategies led to different amounts of underweighting, even when participants saw exactly the same information.

Next, we found that people who switch options frequently, and are thus likely to be using a round-wise strategy (Fig. 3), will generally also be more likely than others to underweight rare events. As already noted, frequent switchers tend to take smaller samples, so they may often underweight rare events simply because they do not experience them. However, even when rare events are experienced, a round-wise strategy may still give rise to underweighting. To evaluate this possibility, we simulated decisions based on the round-wise strategy for frequent switchers and decisions based on the summary strategy for infrequent switchers. Then, we determined how often the predicted decisions were consistent with underweighting rare events. Figure 5a shows that the predicted average proportion of choices consistent with underweighting for frequent switchers using the round-wise strategy would be .65 , and the corresponding proportion for infrequent switchers using the summary strategy would be $.47, t(223.2)=4.8, p<.001$.

Figure $5 \mathrm{~b}$ compares these predicted choices with the choices actually made. The actual choices of frequent switchers were more often consistent with underweighting rare

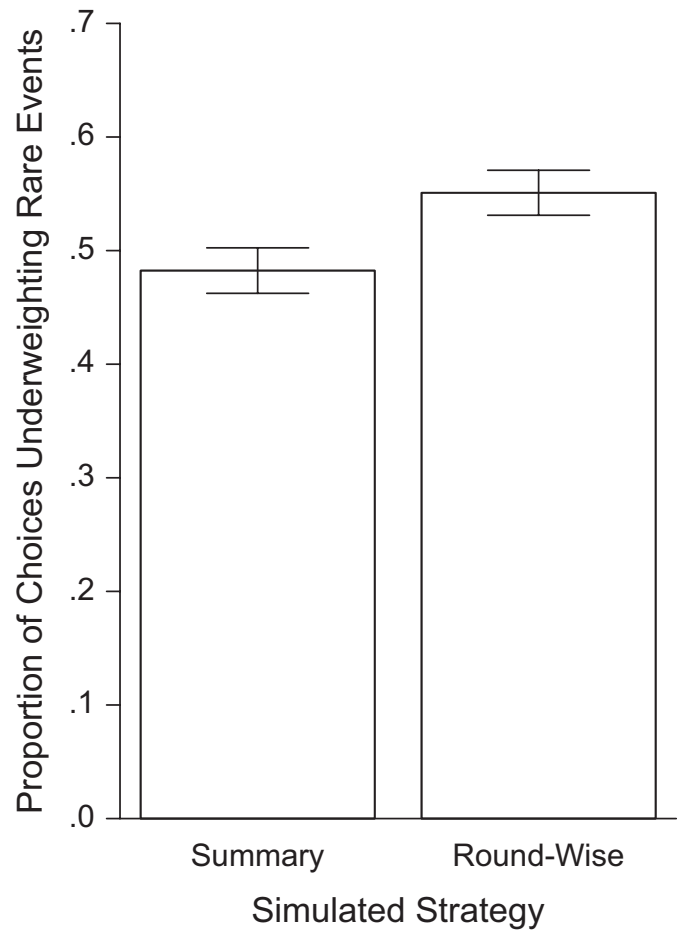

Fig. 4. Underweighting of rare events as a function of decision strategy. On the basis of each participant's sampled outcomes, we simulated decisions assuming a round-wise strategy or a summary strategy. Results were averaged first within participants and then across participants; all 233 participants and their decisions were included in the analysis of each strategy. Error bars represent standard errors of the mean. 
events than were the actual choices of infrequent switchers $(.60$ vs. .52$), t(228.5)=-1.99, p<.05$. Moreover, the observed values were not significantly different from their corresponding predicted values $(p>.05)$. Finally, Figure $5 \mathrm{~b}$ also presents the appropriately weighted mean proportion of decisions from description (taken from all four studies) consistent with underweighting for the same problems. Relative to the decisions of both frequent and infrequent switchers, people's decisions from description were even less consistent with underweighting rare events $(M=.42), t(113)=4.36, p<.001$.

\section{Conclusions}

In decisions from experience, people are the masters of their information search. That is, people control how they distribute their attention over options and for how long they do so. Previous research has focused on the limited size of samples that people have taken before making decisions from experience (e.g., Fox \& Hadar, 2006; Hertwig et al., 2004; Hertwig \& Pleskac, 2010). In this study, we showed that how people search also matters. People's sampling patterns-predominantly piecewise or comprehensive - were indicative of their decisions. Moreover, sampling patterns foreshadowed the impact of rare events - with piecewise sampling often preceding the underweighting of rare events.

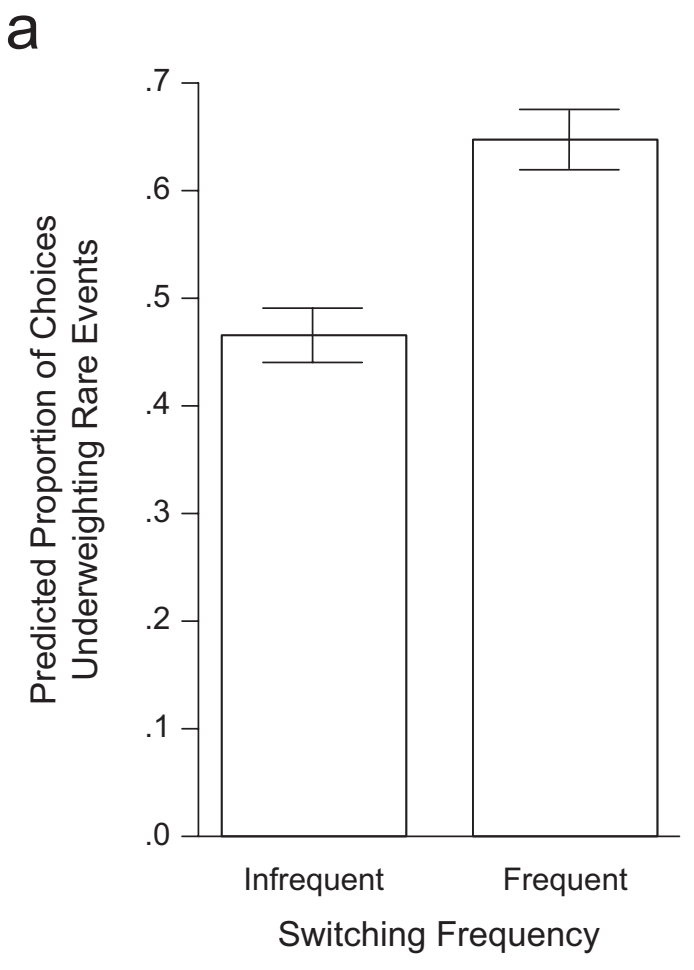

Why is the way people search indicative of the decisions they make? Is it because people initially choose a decision strategy that, in turn, fosters a particular search strategy? Or is it that people at first choose a search strategy, which then leads them to evaluate information in a certain way? Currently, we cannot answer these questions conclusively. It is important, however, to note that piecewise and comprehensive exploration strategies resonate with short-term and long-term exploitation strategies. Key normative and descriptive theories of choice, such as expected value and expected utility, are long term in nature because they recommend choosing the option that is best in the long run (and possibly involves a rare but significant event). Short-term strategies, in contrast, favor options that provide the best outcomes most of the time and are thus more likely to be rewarding in the present. Such strategies are prevalent both theoretically and practically. For example, risk sensitivity and state-dependent foraging explain why a bird that needs food regularly should concern itself with short-term outcomes and not fixate on the long-term average (e.g., Caraco, 1980; Houston \& McNamara, 1999; Stephens, 1981). Similar trade-offs between short-term and long-term outcomes are extremely common, and are evident, for example, in the St. Petersburg paradox (Bernoulli, 1738/1954) and the Iowa gambling task (Bechara, Damasio, Damasio, \& Anderson, 1994). Thus, we speculate that short-term and long-term exploitative

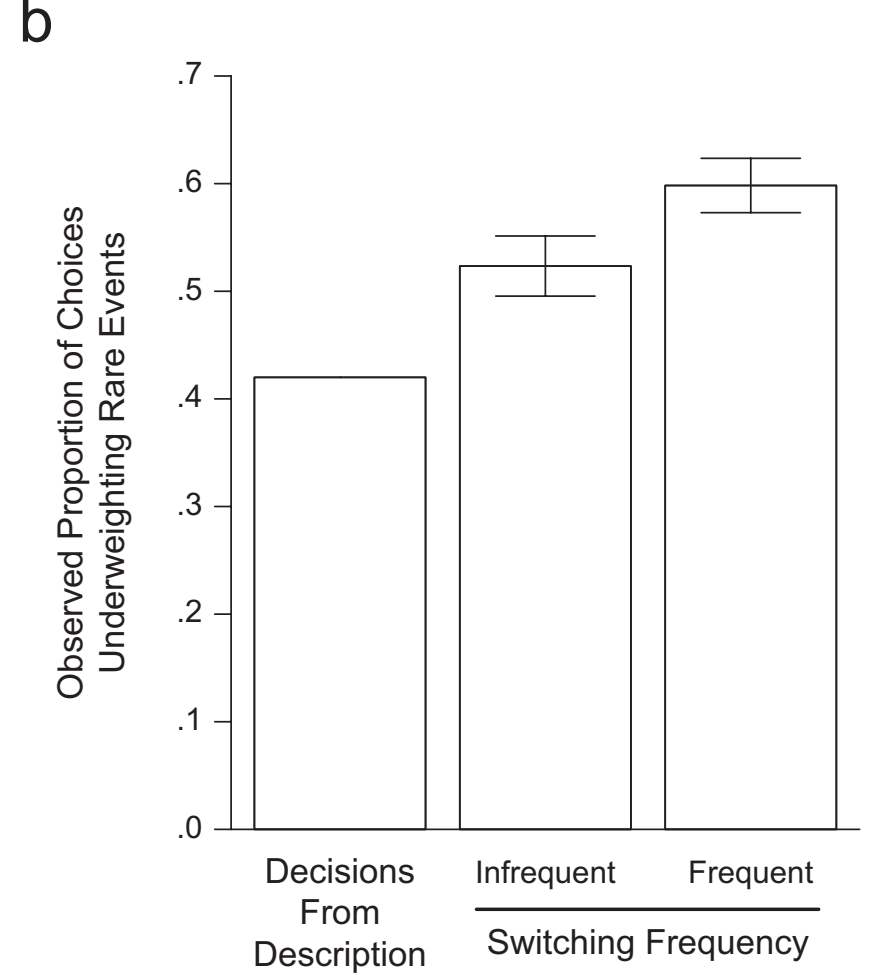

Fig. 5. Predicted and observed proportions of decisions consistent with underweighting rare events among infrequent switchers and frequent switchers. The predicted proportions if all infrequent switchers were employing the summary strategy and all frequent switchers were employing a round-wise strategy are shown in (a). The observed proportions, along with the results observed in the corresponding decisions-from-description conditions, are shown in (b). For decisions from description, we weighted each problem by the number of participants responding in the original studies and by the number of times a problem was represented in the sampling task. Error bars represent standard errors of the mean. 
strategies may to some extent provide a blueprint for exploratory search strategies and the decisions that follow them.

\section{Acknowledgments}

We thank Christoph Ungemach for providing us with data and Laura Wiles for editing the manuscript.

\section{Declaration of Conflicting Interests}

The authors declared that they had no conflicts of interest with respect to their authorship or the publication of this article.

\section{Funding}

This research was supported by grants from the Swiss National Science Foundation to the first author (100014 130397/1) and the second author (100014 126558/1).

\section{Notes}

1. When a round encompassed more than one outcome per option, we computed the mean reward (the sum of outcomes divided by the number of draws; Sutton \& Barto, 1998) per round, per option, to determine the better option for each round. The option that won more rounds was the winner. Winners could also be determined using overlapping rounds (e.g., A-B, B-A, A-B) or a maximax heuristic (comparing the best outcomes from each option per round). These methods generate qualitatively similar results and do not affect our conclusions. The summary strategy compared the overall mean reward for each option. 2. Hertwig et al. (2004), Hau et al. (2008), and Ungemach et al. (2008) used the same 6 decision problems. Hertwig and Pleskac (2010) used 12 problems.

3. We also conducted a multiple regression analysis predicting the proportion of decisions consistent with a round-wise strategy (as in Fig. 1) using both total sample size and switching frequency as predictors. Total sample size was not a significant predictor, $\beta=$ $0.02, t(111)=0.14, p=.89$, but switching frequency was, $\beta=0.41$, $t(111)=2.83, p<.01$.

\section{References}

Bechara, A., Damasio, A.R., Damasio, H., \& Anderson, S.W. (1994). Insensitivity to future consequences following damage to the human prefrontal cortex. Cognition, 50, 7-15.
Bernoulli, D. (1954). Exposition of a new theory on the measurement of risk (L. Sommer, Trans.). Econometrica, 22, 23-36. (Original work published 1738)

Caraco, T. (1980). On foraging time allocation in a stochastic environment. Ecology, 61, 119-128.

Connor, J.A. (2004). Kepler's witch: An astronomer's discovery of cosmic order amid religious war, political intrigue, and the heresy trial of his mother. San Francisco, CA: HarperCollins.

Fox, C.R., \& Hadar, L. (2006). "Decisions from experience" = sampling error + prospect theory: Reconsidering Hertwig, Barron, Weber, \& Erev (2004). Judgment and Decision Making, 1, 159-161.

Hau, R., Pleskac, T.J., Kiefer, J., \& Hertwig, R. (2008). The description-experience gap in risky choice: The role of sample size and experienced probabilities. Journal of Behavioral Decision Making, 21, 493-518.

Hertwig, R., Barron, G., Weber, E.U., \& Erev, I. (2004). Decisions from experience and the effect of rare events in risky choice. Psychological Science, 15, 534-539.

Hertwig, R., \& Erev, I. (2009). The description-experience gap in risky choice. Trends in Cognitive Sciences, 13, 517-523.

Hertwig, R., \& Pleskac, T.J. (2010). Decisions from experience: Why small samples? Cognition, 115, 225-237.

Houston, A.I., \& McNamara, J.M. (1999). Models of adaptive behavior: An approach based on state. Cambridge, England: Cambridge University Press.

Kahneman, D., \& Tversky, A. (1979). Prospect theory: An analysis of decision under risk. Econometrica, 47, 263-291.

Rakow, T., Demes, K.A., \& Newell, B.R. (2008). Biased samples not mode of presentation: Re-examining the apparent underweighting of rare events in experience-based choice. Organizational Behavior and Human Decision Processes, 106 , 168-179.

Stephens, D.W. (1981). The logic of risk-sensitive foraging preferences. Animal Behavior, 129, 628-629.

Sutton, R.S., \& Barto, A.G. (1998). Reinforcement learning: An introduction. Cambridge, MA: MIT Press.

Ungemach, C., Chater, N., \& Stewart, N. (2009). Are probabilities overweighted or underweighted when rare outcomes are experienced (rarely)? Psychological Science, 20, 473-479. 